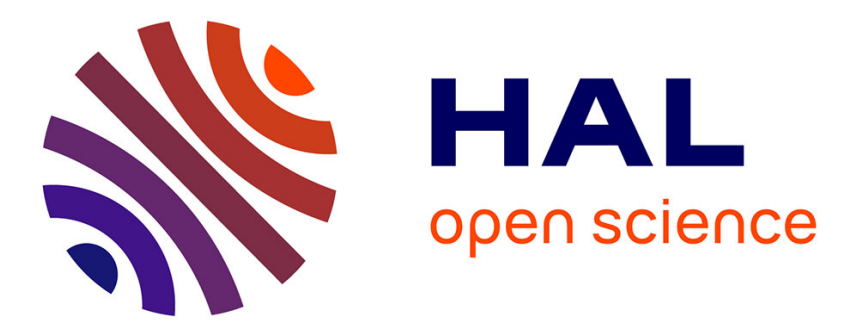

\title{
Propagating double layers in electronegative plasmas
}

\author{
A Meige, Nicolas Plihon, G J M Hagelaar, Jean-Pierre Boeuf, Pascal Chabert, \\ Rod Boswell
}

\section{To cite this version:}

A Meige, Nicolas Plihon, G J M Hagelaar, Jean-Pierre Boeuf, Pascal Chabert, et al.. Propagating double layers in electronegative plasmas. Physics of Plasmas, 2007, 14, pp.053508. 10.1063/1.2736946 . hal-01140488

\section{HAL Id: hal-01140488 \\ https://hal.science/hal-01140488}

Submitted on 9 Apr 2015

HAL is a multi-disciplinary open access archive for the deposit and dissemination of scientific research documents, whether they are published or not. The documents may come from teaching and research institutions in France or abroad, or from public or private research centers.
L'archive ouverte pluridisciplinaire HAL, est destinée au dépôt et à la diffusion de documents scientifiques de niveau recherche, publiés ou non, émanant des établissements d'enseignement et de recherche français ou étrangers, des laboratoires publics ou privés. 


\title{
Propagating double layers in electronegative plasmas
}

\author{
A. Meige ${ }^{a)}$ \\ Laboratoire Plasma et Conversion d'Energie (LAPLACE), Université Paul Sabatier, \\ 118 route de Narbonne, Toulouse Cedex 31062, France and Space Plasma Power and Propulsion Group, \\ Research School of Physical Sciences and Engineering, Australian National University, ACT 0200, \\ Australia
}

N. Plihon ${ }^{\text {b) }}$

Laboratoire de Physique et Technologie des Plasmas (LPTP), Ecole Polytechnique, Palaiseau Cedex 91128, France

G. J. M. Hagelaar and J.-P. Boeuf

Laboratoire Plasma et Conversion d'Energie (LAPLACE), Université Paul Sabatier, 118 route de Narbonne, Toulouse Cedex 31062, France

P. Chabert

Laboratoire de Physique et Technologie des Plasmas (LPTP), Ecole Polytechnique, Palaiseau Cedex 91128, France

R. W. Boswell

Space Plasma Power and Propulsion Group, Research School of Physical Sciences and Engineering, Australian National University, ACT 0200, Australia

(Received 9 February 2007; accepted 30 March 2007; published online 29 May 2007)

\begin{abstract}
Double layers have been observed to propagate from the source region to the diffusion chamber of a helicon-type reactor filled up with a low-pressure mixture of $\mathrm{Ar} / \mathrm{SF}_{6}$ [N. Plihon et al., J. Appl. Phys. 98, 023306 (2005)]. In the present paper the most significant and new experimental results are reported. A fully self-consistent hybrid model in which the electron energy distribution function, the electron temperature, and the various source terms are calculated is developed to investigate these propagating double layers. The spontaneous formation of propagating double layers is only observed in the simulation for system in which the localized inductive heating is combined with small diameter chambers. The conditions of formation and the properties of the propagating double layers observed in the simulation are in good agreement with that of the experiment. By correlating the results of the experiment and the simulation, a formation mechanism compatible with ion two-stream instability is proposed. (C) 2007 American Institute of Physics.
\end{abstract}

[DOI: 10.1063/1.2736946]

\section{INTRODUCTION}

An electric double layer (DL) is a narrow localized region within a plasma, not directly attached to a wall, which can sustain a large potential difference (see Ref. 1, and references therein). Following general terminology, in this paper, the high-potential region is referred to as the "upstream" region and the low-potential region across the DL as the "downstream" region. Double layers have been found in a variety of laboratory plasmas such as in constricted plasmas, ${ }^{2}$ Mercury discharges, ${ }^{3} Q$-machines, ${ }^{4}$ triple plasma devices, ${ }^{5}$ expanding plasmas, ${ }^{6}$ etc. Their role in astrophysics is also considerable as they are thought to be present in the magnetosphere and responsible for the acceleration of electrons onto the upper atmosphere, creating the fantastic aurora. ${ }^{7-9}$ Various theories on the formation of solar flares also involve double layers. ${ }^{10}$ It was also proposed that double layers may play a significant role in supplying and accelerating plasma

\footnotetext{
${ }^{a)}$ Now at the Laboratoire de Physique et Technologie des Plasmas (LPTP), Ecole Polytechnique, Palaiseau Cedex 91128, France; Electronic mail: meige@1ptp.polytechnique.fr.

b) Now at the Laboratoire de Physique UMR CNRS 5672, ENS Lyon, 46 Alle d'Italie, 69007 Lyon, France.
}

in magnetic coronal funnels. ${ }^{1,11}$ In most situations in experiment, theory, and simulation, the DL is imposed and an electric current flows through it. Since the (independent and almost simultaneous) work of Charles and Boswell ${ }^{6}$ and Cohen et $a l .{ }^{12}$ there has been considerable interest in the spontaneous formation of double layers in low-pressure plasmas. Such spontaneous electropositive magnetized double layers have been studied experimentally, ${ }^{6,13-16}$ theoretically ${ }^{17}$ and by computer simulation. ${ }^{18-21}$ More recently, spontaneous static ${ }^{22}$ and propagating ${ }^{23}$ double layers in unmagnetized electronegative plasmas have been reported. Propagating double layers in electronegative plasmas are not only an interesting phenomenon in itself, but also, they need to be understood because of the use of electronegative plasmas in microelectronics.

It is now well established that double layers can form in low-pressure electronegative plasmas; ${ }^{24-29}$ however, the physical processes underlying the formation of propagating double layers remain unclear. The primary goal of the present paper is to investigate plasma discharges similar to that of Plihon et al. ${ }^{23}$ so as to shed light on the critical parameters and the conditions of formation of propagating 


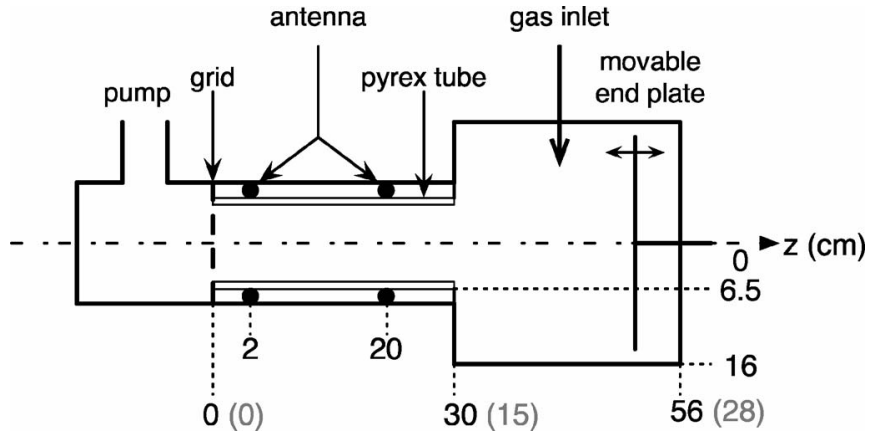

FIG. 1. Schematic of the experimental setup. The dimensions under bracket in gray are that of the corresponding simulation.

electronegative double layers by the use of a computer model. The paper is organized as follows: The most significant and some new experimental results are presented in Sec. II. Section III describes the model. Section IV shows a typical case of spontaneous formation of propagating double layers with the fully self-consistent simulation. Section V presents a parametric study of the propagating double-layer formation. A mechanism for the formation of propagating double layers in electronegative gases is proposed in Sec. VI. The paper is then closed by a few concluding remarks in Sec. VII.

\section{EXPERIMENTAL RESULTS}

Recent experiments by Plihon et al. ${ }^{16,22,23}$ have demonstrated that double layers can form in the expanding region of an inductively coupled electronegative plasma in $\mathrm{Ar} / \mathrm{SF}_{6}$ and $\mathrm{Ar} / \mathrm{O}_{2}$ mixtures. The system is composed of a source chamber, a $30 \mathrm{~cm}$ long, $15 \mathrm{~cm}$ diameter pyrex tube and surrounded by a double-saddle helicon antenna; ${ }^{30}$ the source is attached to a $26 \mathrm{~cm}$ long, $32 \mathrm{~cm}$ diameter aluminum diffusion chamber. A schematic of the setup is shown in Fig. 1. The helicon antenna is powered by an rf power supply operating at $13.56 \mathrm{MHz}$ and capable of delivering $2 \mathrm{~kW}$ of forward power in capacitive or inductive modes.

Plasma parameter measurements reported here were made along the revolution axis ( $z$ axis) of the discharge using electrostatic probes and probe-based laser induced photodetachment. Plasma potential, electron density, and electron temperature were deduced from the $I(V)$ characteristics of a passively compensated Langmuir probe ${ }^{31,32}$ with a $0.25 \mathrm{~mm}$ diameter, $6 \mathrm{~mm}$ long platinum wire tip and from usual $I-V$ curve processing. ${ }^{33}$ A time-resolved setup was developed in order to capture pseudoperiodic regimes, such as propagating double layers. ${ }^{23}$

Measurements of the negative ion density are achieved using probe-based laser induced photodetachment, by measuring the electron current increase following the detachment of electrons induced by collisions between photons and negative ions. ${ }^{34}$ The electronegativity $\alpha$, used throughout the text, is defined as the ratio of the negative ion density $n_{-}$over the electron density $n_{e}$. Time-resolved photodetachment signals were obtained by triggering the frequency tripled $\mathrm{Nd}$ :YAG laser pulse on a delayed double-layer birth signal (the laser pulse period remaining in the range $10 \mathrm{~Hz} \pm 0.1 \mathrm{~Hz}$ ). Probing one period of the phenomenon is made possible by the modification of the delay. Averaging over 200 laser pulses leads to a typical laser induced photodetachment signal.

Plihon et al. ${ }^{16,22}$ showed that a static double layer is formed in the vicinity of the expanding region of the system $(z \sim 30 \mathrm{~cm})$ for moderate electronegativities, i.e., for a rather narrow range of $\mathrm{SF}_{6}$ concentrations (between $8 \%$ and 13\%) and for $\mathrm{O}_{2}$ concentrations above $72 \%$ and for a neutral gas pressure of 1 mTorr. For low electronegativities, no double layer is observed.

At high electronegativities, i.e., in $\mathrm{Ar} / \mathrm{SF}_{6}$ mixtures with $\mathrm{SF}_{6}$ concentrations above $13 \%$, propagating double layers are observed. A full parametric investigation of the periodic formation of propagating double layers is presented in Ref. 23. As shown in the plasma potential measurements in Fig. 2(a), the propagating double layers were born in the vicinity of the interface between the two chambers $(z \sim 26 \mathrm{~cm})$ and propagate downwards, in the diffusion chamber. Note that this regime is periodic-like, with a $5 \%$ variation on the period width. The speed and frequency of these propagating DLs are such that irrespective of the parameters, the number of DLs simultaneously present in the system is constant. The speed of propagation is of the order of $150 \mathrm{~m} / \mathrm{s}$ (which is much slower than the ion sound speed under present conditions) and the frequency in the $\mathrm{kHz}$ range. These propagating double layers share many features with the downstream in-
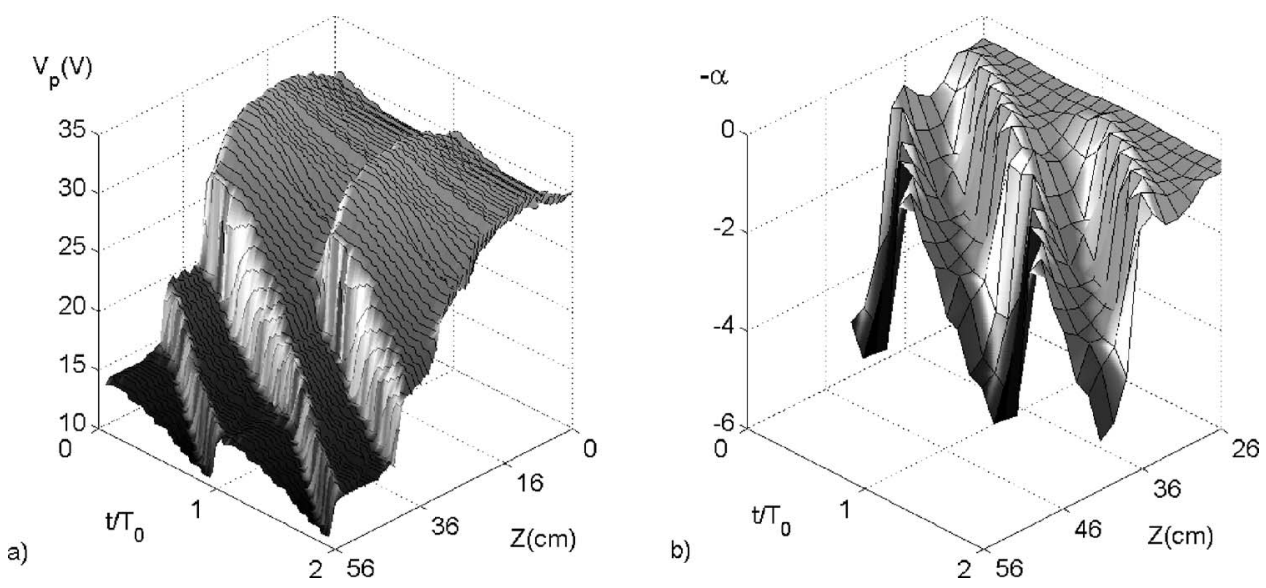

FIG. 2. Spatiotemporal evolution of (a) the plasma potential and (b) the electronegativity in the experimental setup. Time is normalized to the instability period $(\sim 850 \mathrm{~ms})$ for a $25 \%$ $\mathrm{SF}_{6}$ concentration plasma at $600 \mathrm{~W}$ and $1 \mathrm{mTorr}$. 

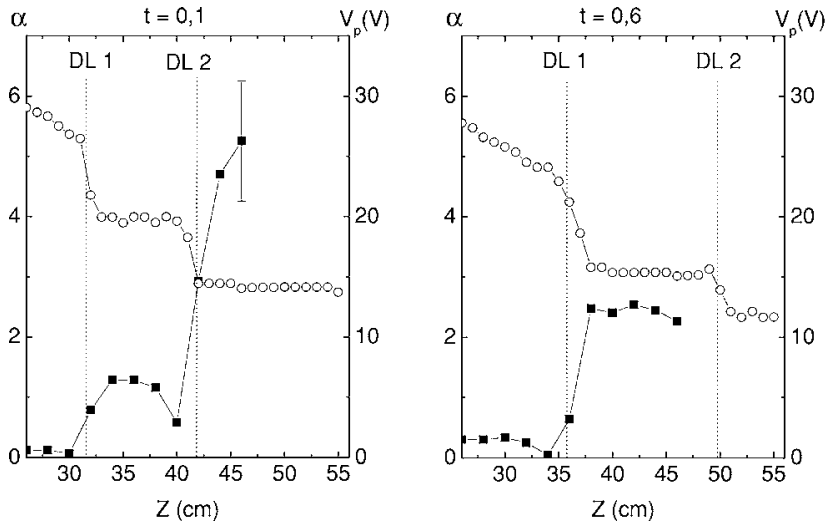

stabilities observed in Refs. 35 and 36 and that were shown to appear when positive and negative ions develop sufficiently large relative drift velocities in the downstream region. ${ }^{37}$

Figure 2(b) shows the time- and space-resolved electronegativity in the diffusion chamber of the experimental setup for the same parameters as in Fig. 2(a). As for the static case, ${ }^{16,22}$ the propagating double layer separates a low electronegativity plasma upstream from a high electronegativity plasma downstream. The correlation between the spatiotemporal plasma potential evolution and the electronegativity is shown in Fig. 2. Figure 3 displays three successive temporal snapshots and shows (i) the propagation of the double layers towards the bottom of the diffusion chamber, (ii) the high correlation between the plasma potential and electronegativity evolutions, and (iii) the increase of electronegativity downstream a double layer as time evolves (as observed downstream of DL1). According to laser induced photodetachment, the electronegativity in the source is very low (less than 0.5; however, no measurements were possible below $26 \mathrm{~cm}$ ), and gradually increases from 1 to 5 downstream of the double layers. One should note that the errors bars displayed in Fig. 3 only accounts for the uncertainty on the electron current increase; some additional error sources (such as the fact that $\mathrm{SF}_{5}^{-}$is not photodetached) may lead to an underestimation of the electronegativity up to a factor of $2 .{ }^{16}$

\section{MODEL}

The simulation of electronegative double layers and more particularly the simulation of propagating double layers is rather challenging; the challenge arises from various things such as the rather high plasma densities, the disparity between the negative ion and electron densities, the long time-scale oscillations, the necessity of self-consistency, etc. Under such conditions, the use of a full particle-in-cell (PIC) simulation would be too computationally expensive, while the use of a "classical" hybrid PIC-Boltzmann model would not be appropriate as it is not completely self-consistent; hence, we have developed the "improved" hybrid PICBoltzmann simulation $\mathrm{h} 2 \times$ presented below.

\section{A. "Classical" hybrid model}

Our $\mathrm{h} 2 \times$ simulation is based on the "classical" hybrid following the same basic algorithm as a standard particle-in-

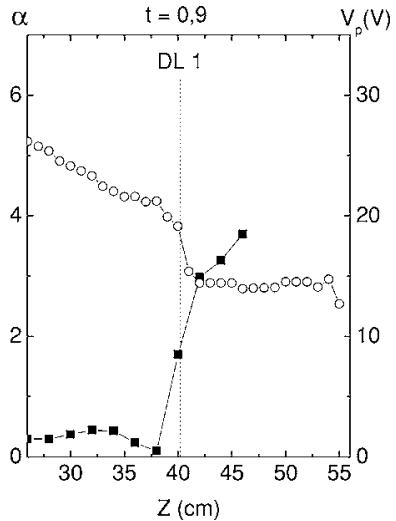

FIG. 3. Spatial evolution of the electronegativity ( $\mathbf{\square})$ and plasma potential $(\bigcirc)$ at three different instants during one period of the instability (time normalized to the instability period). Same conditions as Fig. 2.

cell simulation ${ }^{38,39}$ and is described in detail in Ref. 40. In short, (i) the charges are accumulated on the mesh, (ii) Poisson's equation is solved to find the corresponding electric field, and (iii) Newton's law is used to accelerate the particles according to the electric field. These steps constitute one iteration and iterations are repeated until the simulation reaches steady state. In addition, the electrons are assumed to be in Boltzmann equilibrium and their density $n_{e}$ is given by

$$
n_{e}=n_{0} \exp \frac{e \Phi}{k_{\mathrm{B}} T_{e}},
$$

where $n_{0}$ is the electron density at the point where the potential $\Phi$ is null, $T_{e}$ is the electron temperature, and $k_{\mathrm{B}}$ is the Boltzmann constant. Assuming Boltzmann electrons a priori allows the simulation to "jump over" the plasma frequency. This permits the use of much larger time steps than with the full classical particle-in-cell scheme, significantly reducing computational cost and therefore time to convergence.

Poisson's equation is coupled with the Boltzmann relation in the following way. Let the upper indices refer to a moment in time and let $\Delta t$ be a time step, with $t^{k+1}=t^{k}+\Delta t$. Assuming that the value of the quantities are known at $t^{k}$ and are to be calculated at $t^{k+1}$.

Poisson's equation reads

$$
\left(\frac{\partial^{2} \Phi}{\partial x^{2}}\right)^{k+1}=-\frac{\rho}{\varepsilon_{0}}=-\frac{e}{\varepsilon_{0}} t\left(n_{+}^{k}-n_{-}^{k}-\widetilde{n}_{e}^{k+1}\right),
$$

where $n_{+}^{k}$ and $n_{-}^{k}$ are, respectively, the positive and negative ion densities coming from the accumulation of the particle ion charges on the mesh. To avoid numerical instabilities, a first-order estimate of the electron density $\tilde{n}_{e}^{k+1}$ (function of $\Phi^{k}$ and $\Phi^{k+1}$ ) at $t^{k+1}$ is used. The spatial integration of Eq. (2) is performed following a classical algorithm for solving tridiagonal systems (Ref. 41, for example). In hybrid models, the electron density reference $n_{0}$ is commonly imposed. Imposing $n_{0}$ can lead to a miscalculation of the sheath potential and to errors in the calculation of plasma parameters. In the present work, the density reference $n_{0}$ is self-consistently calculated at every time step; this is done by estimating the real electron flux to the walls and an electron balance within the plasma (accounting for electron loss and creation). Details for the calculation of $n_{0}$ can be found in Refs. 40 and 42. 


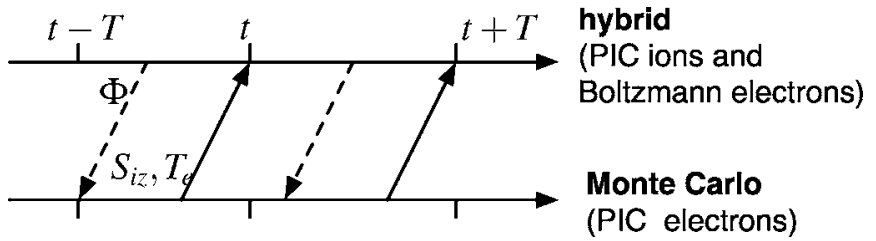

FIG. 4. The model $\mathrm{h} 2 \times$ (hybrid $2 \times$ ) is composed of two submodels coupled to each other, allowing to self-consistently simulate low-pressure high-density plasmas.

\section{B. Self-consistent calculations of the electron energy and source term profiles: Model h2X}

"Classical" hybrid models, such as that presented in Sec. III A, are widely used in many fields of plasma physics, such as plasma propulsion, ${ }^{43}$ plasma processing, ${ }^{44,45}$ fundamental plasma physics, ${ }^{28}$ etc. These models provide a complete description of the discharge, at relatively low computational cost, using the Boltzmann relation to describe the electron transport, coupled to Poisson's equation to describe the electric field. However, unlike full particle-in-cell simulations, hybrid models are based on restrictive assumptions for the electron energy distribution function (EEDF), as it is assumed that electrons have a Maxwell-Boltzmann distribution with a specific temperature, thus failing (i) to account for possible more complex distributions, for example having an overpopulated group of low energy electrons or a depleted tail and (ii) to self-consistently calculate the creation source term profiles.

In the following, we show how to self-consistently calculate the electron temperature ${ }^{51}$ and the source term profile by coupling a Monte Carlo model for particle electrons to the classical hybrid model. In the following, the novel model shall be called $\mathrm{h} 2 \times$, standing for hybrid $2 \times$, as it is a hybrid PIC-Boltzmann-Monte Carlo.

As shown in Fig. 4, the general concept of $\mathrm{h} 2 \times$ is to have two submodels coupled to each other, each of them using the results of the other as input parameters. Iterations between the two parts of the model are done until the "steady state" is reached. Each part of the model transfers its results to the other part at a regular period $T$, a parameter of the simulation. (i) The first submodel is a classical hybrid model where the ions are treated as particles while electrons obey the Boltzmann relation and determine the plasma potential. (ii) The second submodel is a Monte Carlo for particle electrons that calculates the electron temperature and the ioniza- tion source term. The electron temperature used in the hybrid submodel is the spatial average of the spatially dependent temperature calculated by the Monte Carlo.

The Monte Carlo submodels also incorporates a heating mechanism similar to that described in Ref. 19. In short, an ac electric field perpendicular to the spatial dimension of the simulation heats the electrons. This scheme is intended to model inductive heating without solving electromagnetism equations. In addition, particle radial losses may play a significant role in discharges whose diameter is small compared to their length. Hence, a volumic loss mechanism is also included in the Monte Carlo submodel. Electrons whose perpendicular energy $\mathcal{E}_{\perp}$ is greater than the local plasma potential $\Phi$ have a certain probability to be lost radially. The loss probability for a discharge of radius $R$, given in terms of a loss frequency, is $\nu_{e, \text { loss }}=\left|v_{\perp} / R\right|$, if $\mathcal{E}_{\perp}>\Phi$ and $\nu_{e, \text { loss }}=0$, otherwise. Note that $v_{\perp}$ is the electron velocity in the direction perpendicular to the spatial dimension of the simulation.

\section{The third species}

A very simplified mixture of argon and $\mathrm{SF}_{6}$ is to be simulated. Only one type of negative ion with the same mass as the argon positive ion and with an opposite charge $-e$ is considered. Due to the presence of the third species, namely, the negative ions, a number of events, such as attachment and recombination $\left(\mathrm{SF}_{6}\right.$ is recombination dominated, hence detachment is not considered), have to be treated in addition to ionization. Simplified attachment cross sections based on that of $\mathrm{SF}_{6}$ compiled by Phelps and van Brunt ${ }^{46}$ were used. Figure 5(a) shows the electron-neutral collision cross sections in $\mathrm{SF}_{6}$, including elastic collisions (i), ionization (h), excitation $(\mathrm{d}-\mathrm{g})$ and attachment $(\mathrm{a}-\mathrm{c})$. Figure 5(b) shows the attachment cross sections in $\mathrm{SF}_{6}$ and the simplified attachment cross sections that were used for the present simulations. In addition to undergoing collisions with argon neutral atoms, electrons can also undergo collisions with neutral $\mathrm{SF}_{6}$; the cross section corresponding to these collisions is shown by line (i) in Fig. 5(a). The other exciting and ionizing collisions with $\mathrm{SF}_{6}$ are not considered. In the following, the simplified electronegative background gas is loosely called $\mathrm{SF}_{6}$. Positive and negative ions recombine with a rate $K_{\text {rec }}$ $=10^{-13} \mathrm{~m}^{3} \mathrm{~s}^{-1}$, unless stated otherwise.

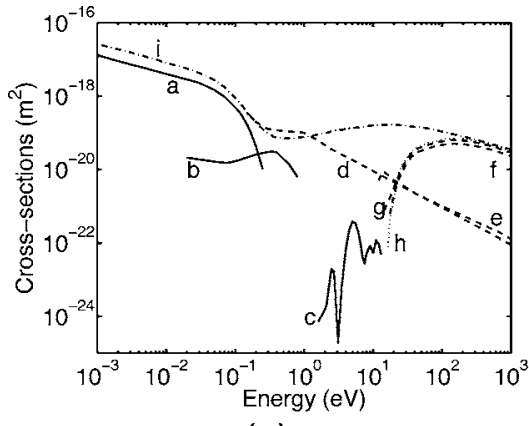

(a)

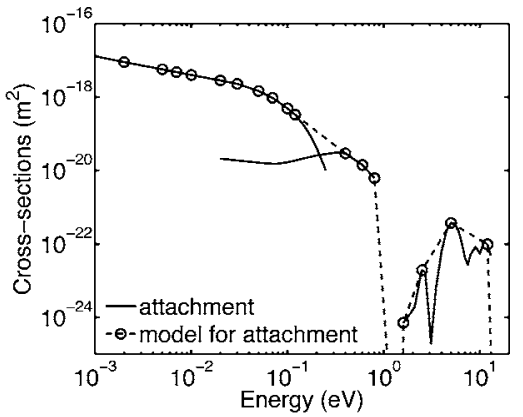

(b)
FIG. 5. (a) Electron-neutral collision cross section with $\mathrm{SF}_{6}$; attachment: $(\mathrm{a}-\mathrm{c})$; excitations: $(\mathrm{d}-\mathrm{g})$; ionizations: (h); elastic collisions: (i). (b) Attachment cross section in $\mathrm{SF}_{6}$ (solid lines) and simplified total attachment cross-section used in the simulation (broken line). 
TABLE I. General parameters of the one-dimensional simulation of spontaneous formation of propagating double layers. These parameters were used in the present investigation, unless stated otherwise.

\begin{tabular}{ll}
\hline \hline Quantity & Value \\
\hline System length & $28 \mathrm{~cm}$ \\
Heating length & $15 \mathrm{~cm}$ \\
System radius & $10 \mathrm{~cm}$ (uniform) \\
Cell number & 70 \\
Total duration & $10-100 \mathrm{~ms}$ \\
Hybrid time step & $5 \times 10^{-8} \mathrm{~s}$ \\
Monte Carlo time step & $5 \times 10^{-10} \mathrm{~s}$ \\
Macroparticle weight & $1 \times 10^{14} \mathrm{~m}^{-3}$ \\
Positive and negative ion mass (Ar) & $6.68 \times 10^{-26} \mathrm{~kg}$ \\
Positive and negative ion charge & $+e$ and $-e$ \\
Room temperature & $297 \mathrm{~K}^{21}$ \\
Source term & $4 \times 10^{21} \mathrm{~m}^{-3} \mathrm{~s}^{-1}$ \\
Total neutral pressure & $1 \mathrm{mTorr}^{-13} \mathrm{~m}^{3} \mathrm{~s}^{-1}$ \\
$K_{\text {rec }}$ & $10^{-1}$ \\
\hline \hline
\end{tabular}

\section{FULLY SELF-CONSISTENT DOUBLE-LAYER SIMULATION}

Using the fully self-consistent model above, with the general parameters given in Table I and a relative $\mathrm{SF}_{6}$ concentration of $15 \%$, a high-density plasma sustaining spontaneous propagating double layers was simulated. Propagating double layers were only observed for sufficiently small chamber radii (typically less than $20 \mathrm{~cm}$ ) and inductive heating localized to the source region $(0-15 \mathrm{~cm})$.

Figure 6(a) shows a snapshot of the plasma potential profile as a function of position; in addition to the smooth decrease of the potential from the source to the diffusion chamber, a series of potential "steps" and a clear potential drop of $\sim 5 \mathrm{~V}$ are observed in the downstream region. This sudden potential drop is extremely sharp and occurs within less than $\sim 1 \mathrm{~cm}$. Figure 6(b) shows that the potential drop is accompanied by an electron temperature drop; the high and low-potential side temperatures are $\sim 5$ and $\sim 3.5 \mathrm{eV}$, respectively, which is in good agreement with that experimentally measured by Plihon et al. ${ }^{22}$ As explained later in Sec. VI, the temperature drop observed in both the simulation and the experiment is attributed to a combination of the localized heating and the electron radial loss.

Figure 6(c) shows the electron (solid line), positive (dashed line), and negative (dotted-dashed line) ion densities. A series of spatial oscillations in both positive and negative ion densities are observed. These oscillations are successive regions of rarefaction of positive and negative ions. Each of the ion density local minima corresponds to a plasma potential "step" [Fig. 6(a)]. The electronegativity $\alpha$ is around 1 in the source region, as high as $\sim 8$ between two successive double layers, and reaches a local minimum at the position of each double layer. Note that the plasma is observed to be stratified; i.e., the negative ions are confined to the core of the plasma and their density drops to zero before the sheaths. ${ }^{47}$

Finally, Fig. 6(d) shows the plasma potential resolved in space (horizontal axis) and time (vertical axis). Five potential fronts, corresponding to five double layers are observed and are propagating towards the source region. A time-resolved analysis of the densities also shows that the spatial oscillations mentioned before propagate towards the source region, following the potential drops.

By using the fully self-consistent simulation, propagating double layers were only observed under the following conditions: (i) inductive heating mechanism localized to the source region (from 0 to $15 \mathrm{~cm}$ ), (ii) small chamber radius

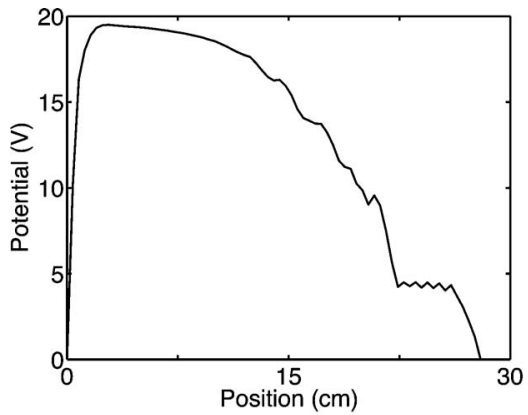

(a)

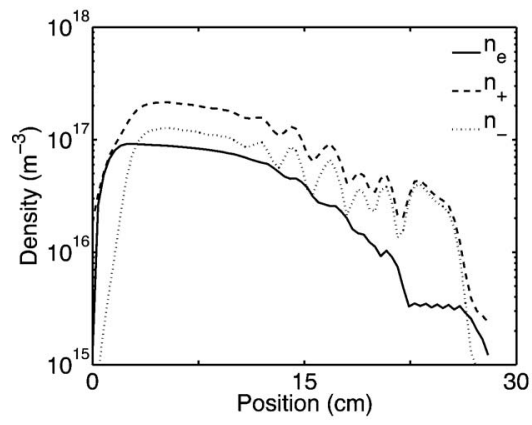

(c)

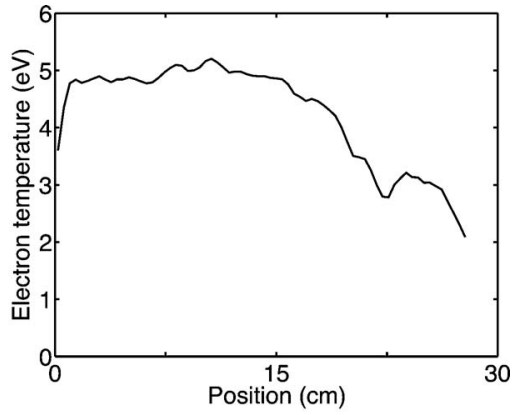

(b)

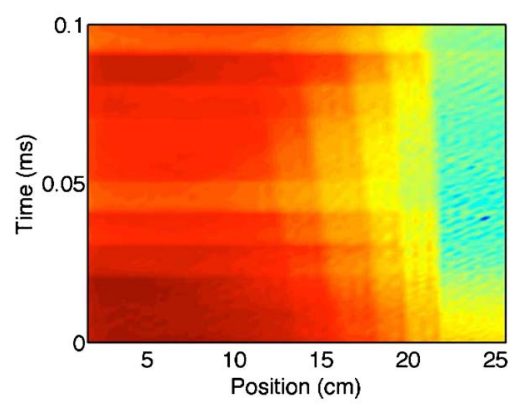

(d)
FIG. 6. (Color online) Self-consistent spontaneous formation of propagating double layers in an electronegative discharge. (a) Snapshot of the plasma potential profile presenting a clear drop of $\sim 5 \mathrm{~V}$; (b) snapshot of the corresponding electron temperature profile, also presenting a drop; (c) snapshot of the electron (solid line), positive ion (dashed line) and negative ion (dotteddashed line) density profiles; (d) plasma potential as a function of space and time when the electronegative propagating double layers are formed. 
( $\$ 20 \mathrm{~cm}$ ), (iii) sufficiently high $\mathrm{SF}_{6}$ concentration $(\gtrsim 15 \%)$, and (iv) low gas pressure ( $\leqslant 5 \mathrm{mTorr}$ ). Static electronegative double layers similar to those of Plihon et al. ${ }^{22}$ were not found in the simulation.

\section{PARAMETRIC STUDY OF DOUBLE LAYERS}

For the sake of simplicity, rather than coupling the two submodels as was previously done, a temperature profile $T_{e}(x)$ similar to that obtained self-consistently is now used as a parameter of the simulations (the electron Monte Carlo is deactivated) in order to perform a more systematic parametric investigation of the spontaneous propagating double layers. By integrating the ionization and attachment cross sections over a Maxwellian distribution, the fits for the attachment and ionization rate coefficients ${ }^{52}$ and for the corresponding space-dependent ionization and attachment frequencies $\nu_{\mathrm{iz}}\left[T_{e}(x)\right]$ and $\nu_{\mathrm{att}}\left[T_{e}(x)\right]$ are found. The temperature profile decreases smoothly from $4.5 \mathrm{eV}$ in the source to $3 \mathrm{eV}$ in the diffusion chamber. Note that the temperature profile was only used to calculate the various reaction frequencies (ionization and attachment): the average over space of this temperature profile was used in the Boltzmann relation [Eq. (1)].
The effect of almost every parameter such as the positive and negative ion masses, the concentration of $\mathrm{SF}_{6}$, the system dimensions (length and radius), the temperature profile, etc., was investigated; the most relevant are reported here. Numerical parameters, such as the spatial resolution of the grid and the time step, were varied without significantly affecting the results.

\section{A. Double layers form at low pressure with high attachment and recombination}

Figure 7(a) shows a spatiotemporal representation of the plasma potential profiles and Fig. 7(b) shows snapshots of the plasma potential profiles as a function of space for various concentrations of $\mathrm{SF}_{6}$. Propagating double layers do not form unless the relative concentration of $\mathrm{SF}_{6}$ is sufficiently large ( $230 \%$ under the present conditions), confirming that a minimal electronegativity is required. As it was already mentioned, static electronegative double layers similar to that of Plihon et $a .^{22}$ were not found in the simulation.

When the real EEDF is calculated by the simulation (Sec. IV), the minimal relative concentration of $\mathrm{SF}_{6}$ required for the formation of propagating double layers (i.e., to reach sufficient electronegativity) is $\sim 15 \%$. In the present case,

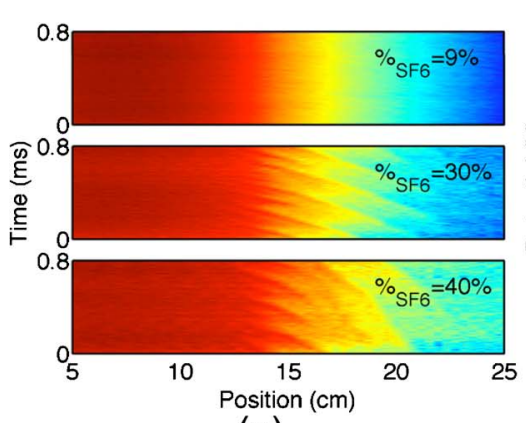

(a)

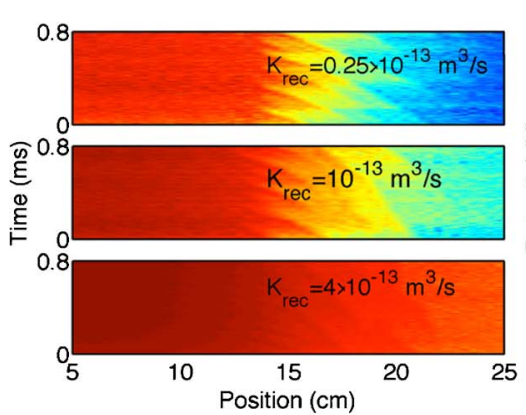

(c)

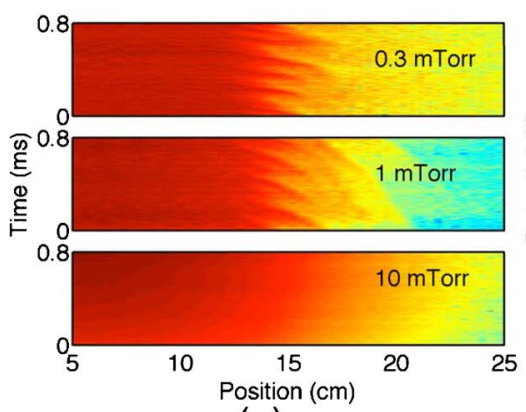

(e)

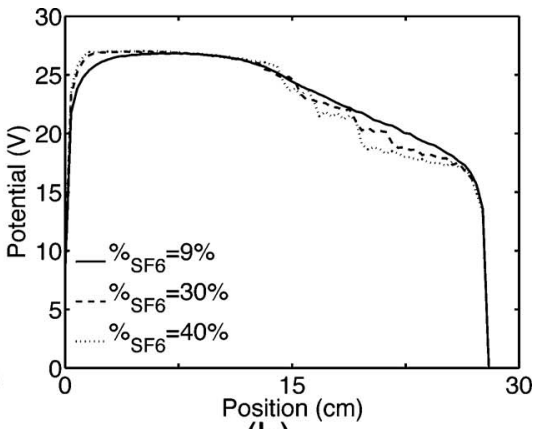

(b)

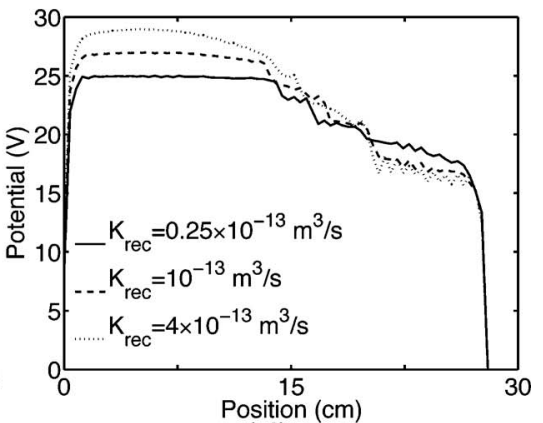

(d)

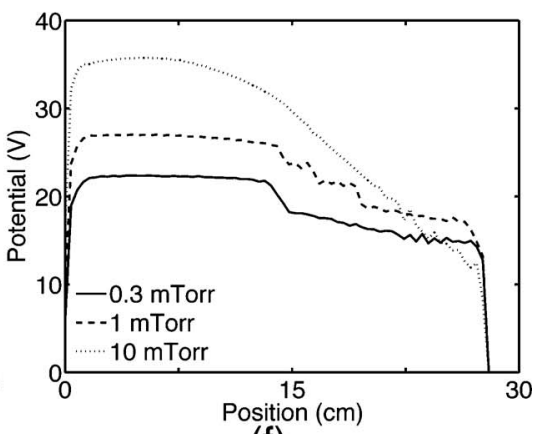

(f)
FIG. 7. (Color online) Contour plots of the plasma potential profiles as a function of space and time, and snapshots of the plasma potential profiles, for various relative concentrations of $\mathrm{SF}_{6}$ [(a) and (b)], recombination rate coefficients [(c) and (d)], and neutral gas pressures [(e) and (f)]. 
when the EEDF is assumed to be Maxwellian, almost twice this concentration $(z 30 \%)$ is required to observe the propagating double layers. A reason for this is presumably that the EEDF calculated by the Monte Carlo submodel was not Maxwellian (high-energy depletion and low-energy population) favoring attachment to ionization, leading to sufficient electronegativity for the formation of propagating double layers. This peculiar EEDF is not captured when assuming Maxwell-Boltzmann electrons; thus, the concentration of $\mathrm{SF}_{6}$ has to be increased accordingly in order to reach sufficient electronegativity, compatible with the double layers. This provides further evidence supporting the fact that the EEDF is a rather important parameter in the formation of electronegative double layers.

Figure 7(c) shows the plasma potential profiles as a function of space and time, while Fig. 7(d) shows snapshots of the plasma potential profiles as a function of space for various recombination rate coefficients $K_{\text {rec }}$. The electronegativity is maintained constant by changing the relative concentration of $\mathrm{SF}_{6}$ accordingly. For the range of $K_{\text {rec }}$ investigated, the double-layer potential drop increases with $K_{\text {rec }}$. For similar electronegativities, propagating double layers are only observed for sufficiently high attachment and recombination. This is in good agreement with previously simulated electronegative double layers. ${ }^{26,28}$
The total neutral gas pressure was varied from 0.1 to 10 mTorr, keeping the relative concentrations of argon and $\mathrm{SF}_{6}$ constant. Figures $7(\mathrm{e})$ and $7(\mathrm{f})$ show that above a critical pressure ( 25 mTorr) no double layer was formed.

To summarize this subsection, it should be remembered that propagating double layers were only observed at low pressure and for sufficiently high electronegativity obtained with high attachment and high recombination. This allows the existence of relatively cold negative ions, compatible with electronegative double layers. ${ }^{28}$

\section{B. Small-diameter and long chambers are propitious to the formation of double layers}

As mentioned in Sec. IV, propagating double layers were only observed when the radius of the chamber was relatively small and when the heating mechanism was localized to the source region. However, the geometric transition between the source region and the diffusion chamber does not seem to be a fundamental parameter in neither the experiment ${ }^{23}$ or the simulation, as in both cases it was possible to form double layers. Nevertheless, the strength of the double layers was observed to be enhanced by the presence of a geometric transition.

Increasing the relative length of the diffusion chamber

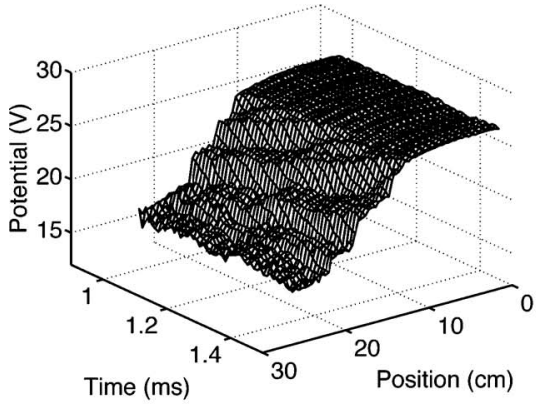

(a)

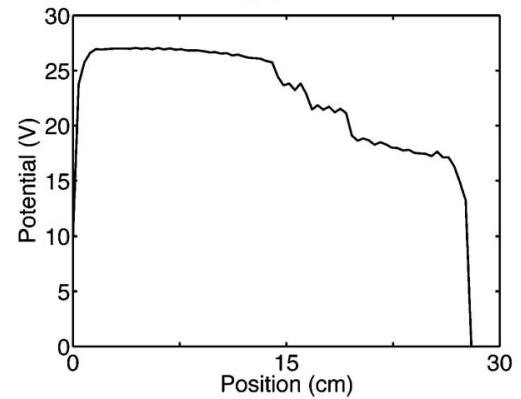

(c)

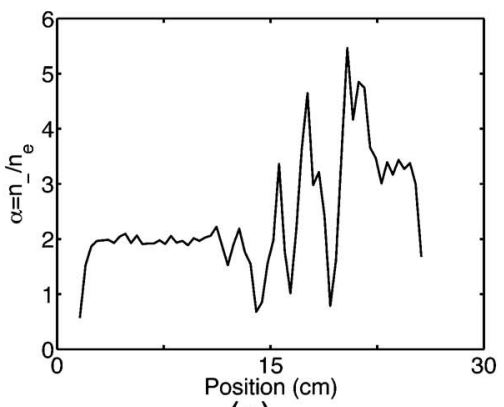

(e)

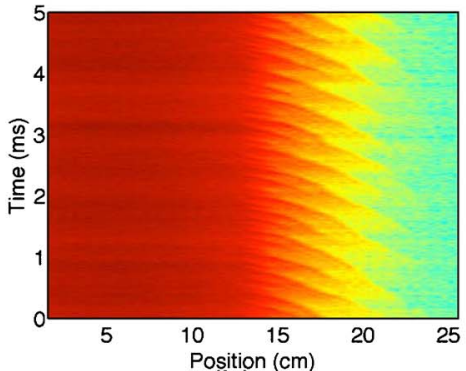

(b)

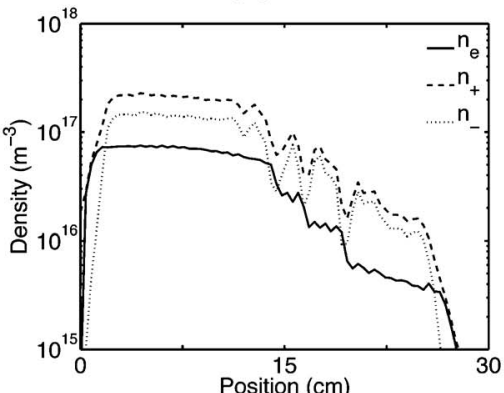

(d)

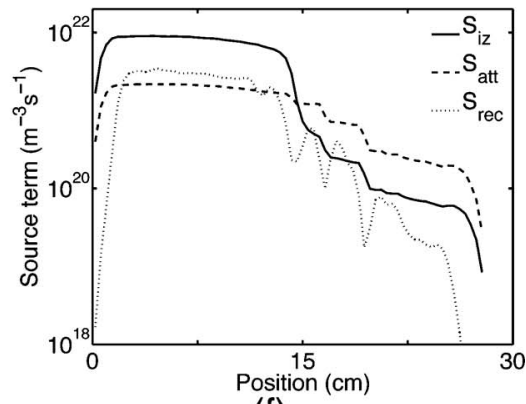

(f)
FIG. 8. (Color online) Propagating double layers in an $\mathrm{Ar} / \mathrm{SF}_{6}$ mixture, with an $\mathrm{SF}_{6}$ relative concentration of $40 \%$ at 1 mTorr. (a) 3D mapping of the plasma potential profile as a function of space and time. (b) Contour plot of the plasma potential profile as a function of space and time, increased brightness indicates decreased potential. (c) Snapshot of the plasma potential profile showing successive double layers, propagating from the diffusion chamber (right) to the source (left). (d) Snapshots of the electron (solid line), positive (dashed line) and negative (dotted-dashed line) ion density profiles. (e) Snapshot of the electronegativity $\alpha$ $=n_{-} / n_{e}$ profile. (f) Snapshots of ionization (solid line), attachment (dashed line) and recombination (dotteddashed line) source term profiles. 
leads to an increase in the number of propagating double layers simultaneously present in the system, while reducing it leads to a decrease of the number of propagating double layers. When the diffusion chamber length is sufficiently decreased, no propagating double layer forms. This is also very much aligned with the experimental results showing that the number of propagating double layers decreases and eventually reaches zero when the movable end plate (Fig. 1) is pushed towards the source.

\section{Characterization of the propagating double layers}

Figure 8 shows various characteristics of typical propagating double layers for an $\mathrm{SF}_{6}$ concentration of $40 \%$. Figure $8(\mathrm{a})$ is a three-dimensional (3D) mapping of the plasma potential as a function of time and space. Although the present double layers propagate in the opposite direction, they appear to be a phenomenon very similar to that observed by Plihon et al. ${ }^{23}$ as discussed later.

Figure 8(b) is a contour plot over $5 \mathrm{~ms}$ of the plasma potential profile. As can be observed, the double layers always form at the same critical position and propagate towards the source at $\sim 100 \mathrm{~m} / \mathrm{s}$. Note that the simulation was run up to $100 \mathrm{~ms}$ without any major variation.

Figures 8(c) and 8(d) are snapshots of the plasma potential and the electron, positive, and negative ion densities. At the position of each double layer, a region of positive and negative ion rarefaction can be observed. Figure 8(e) shows a snapshot of the electronegativity $\alpha=n_{-} / n_{e}$ at the same instant and the electronegativity is minimal at the positions of the double layers, but maximal immediately downstream. The regions of high electronegativity are trapped between two successive double layers, and are pushed towards the source by the moving double layers, which is very much aligned with the experiment, as shown in Fig. 3.

Figure 8(f) shows the ionization (solid line), attachment (dashed line), and recombination (dotted-dashed) source term profiles. The source is ionization-dominated, while the diffusion chamber is attachment dominated. The recombina-

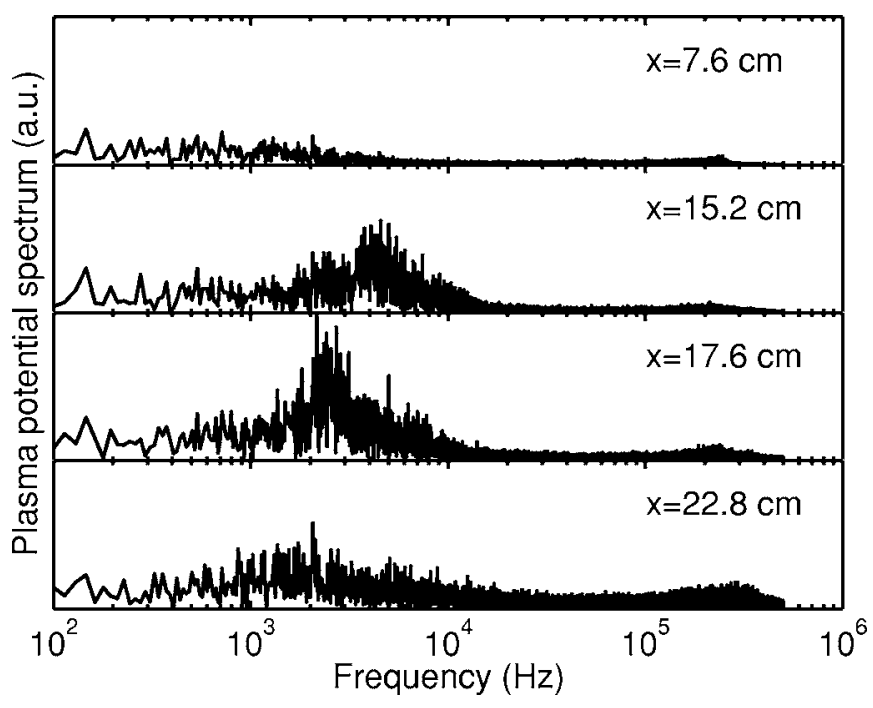

FIG. 9. Frequency spectrum of the plasma potential at various positions in the system.

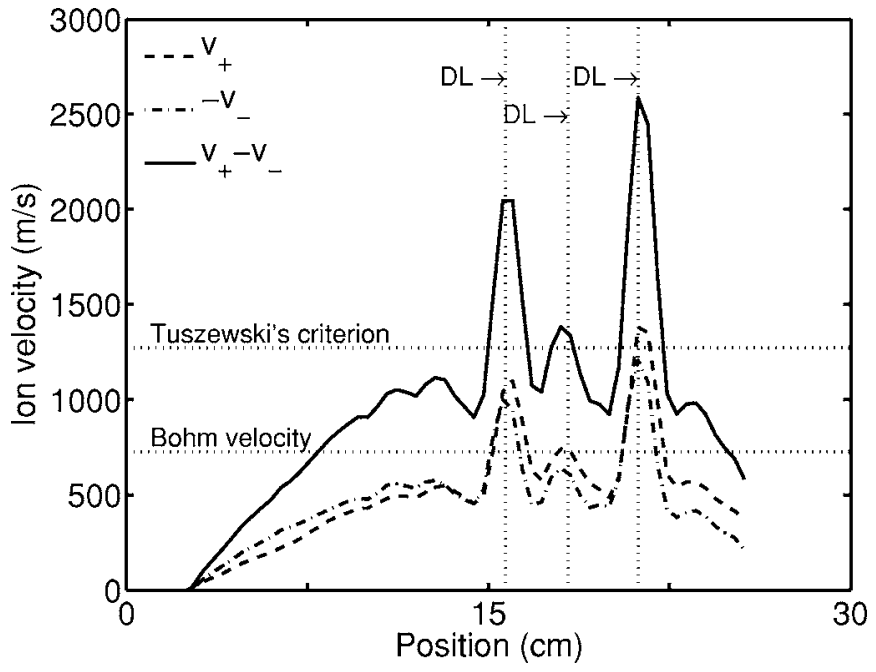

FIG. 10. Snapshot of the positive and negative ion average velocities $v_{+}$, $-v_{-}$(broken lines) and the relative drift velocity $v_{+}-v_{-}$(solid line), as a function of position. The vertical dotted lines show the positions of the double layers, while the horizontal lines show the Bohm velocity and "Tuszewski's criterion."

tion takes place mostly in the source, but also in the highelectronegativity regions, where the ion densities are maximum.

Figure 9 shows a low-frequency activity around $2-4 \mathrm{kHz}$ in the downstream plasma (i.e., where the double layers are observed). This activity corresponds to the train of propagating double layers born at some critical downstream position and propagating towards the source. In the range of pressures investigated, the frequency of the propagating double layers was found to be weakly dependent on the neutral gas pressure.

Figure 10 shows a snapshot of the positive and negative ion average velocities: $v_{+}$and $-v_{-}$. The average downstream ion sound speed is shown by the lower horizontal line. At the positions of the propagating double layers, shown by the vertical lines, both positive and negative ions reach the sound speed (as derived by Braithwaite and Allen ${ }^{48}$ and Franklin and Snell ${ }^{49}$ ) before entering the double layers from the high- and low-potential sides, respectively. The ions are then accelerated through the double layers. This shows that the Bohm criterion is verified for both positive and negative ions at the entrance of each double layer.

In addition, the propagating double layers observed in both the experiment and the simulation share many properties (in the $\mathrm{kHz}$ range, propagation at $\sim 100 \mathrm{~m} / \mathrm{s}$, a few mTorr, etc.) with the downstream instability observed by Tuszewski et al. ${ }^{36}$ and Tuszewski and Gary. ${ }^{37}$ Although they have not explicitly measured a double layer, Tuszewski et $a l .,{ }^{36}$ have observed that the plasma immediately downstream of the critical position, where the downstream instability seems to be born, was transiently electropositive, suggesting the existence of an internal sheath; i.e., a double layer. Tuszewski and Gary ${ }^{37}$ showed that the downstream instability is compatible with a two-stream instability between the positive and negative ions. The upper horizontal line in Fig. 10 shows "Tuszewski's criterion"; i.e., the mini- 
TABLE II. Experimental and simulated propagating double layers.

\begin{tabular}{|c|c|c|}
\hline Parameters & Experiment & Simulation \\
\hline Percent $\mathrm{SF}_{6}$ & $13 \%-25 \%$ & $15 \%^{\mathrm{a}}$ \\
\hline$K_{\mathrm{rec}}$ & Not a parameter & Critical $^{\mathrm{b}}$ \\
\hline$P^{\mathrm{c}}$ & $\sim 5$ mTorr & $\sim 5$ mTorr \\
\hline Expansion & Not critical & Not critical \\
\hline Small diameter & Not known & Critical $^{\mathrm{d}}$ \\
\hline Chamber length $^{\mathrm{e}}$ & Critical & Critical \\
\hline Properties & Experiment & Simulation \\
\hline Frequency & $\sim \mathrm{kHz}$ & $\sim 2 \mathrm{kHz}$ \\
\hline Velocity & $150 \mathrm{~m} / \mathrm{s}$ & $100 \mathrm{~m} / \mathrm{s}$ \\
\hline Direction & $\rightarrow$ & $\leftarrow$ \\
\hline$\alpha^{\mathrm{f}}$ & $\sim 4$ & $4-6$ \\
\hline Potential drop & $7 \mathrm{~V}^{\mathrm{g}}$ & $5 \mathrm{~V}^{\mathrm{h}}$ \\
\hline$T_{\text {up }} / T_{\text {down }}$ & $5 / 3.5 \mathrm{eV}^{\mathrm{i}}$ & $5 / 3.5 \mathrm{eV}$ \\
\hline Bohm criterion $^{\mathrm{j}}$ & Not known & Satisfied \\
\hline
\end{tabular}

${ }^{\mathrm{a}}$ Minimal concentration allowing the formation of double layers in the simulation: $15 \%$ with fully selfconsistent model; $30 \%$ when Boltzmann electrons are assumed.

${ }^{\mathrm{b}}$ The simulation showed that DLs were formed only for sufficiently large recombination rate. DLs were observed in the simulation for realistic recombination rates $\left(K_{\mathrm{rec}}=10^{-13} \mathrm{~m}^{3} \mathrm{~s}^{-1}\right)$.

${ }^{c}$ Pressure $P$ above which no double layer was formed.

${ }^{\mathrm{d}}$ No double layer observed for large-diameter chambers.

${ }^{\mathrm{e}} \mathrm{S}$ Sufficiently short diffusion chambers prevent the formation of double layers.

${ }^{\mathrm{f}}$ Maximum of the electronegativity $\alpha$ obtained just downstream of the double layers. Measured via photodetachment for the experimental values. The error bar is $+100 \%$ and $-20 \%$ for the experimental data.

${ }^{8} 7 \mathrm{~V}$ at the potential discontinuity, almost $10 \mathrm{~V}$ in total.

${ }^{\mathrm{h}} 5 \mathrm{~V}$ with the fully self-consistent model, $2 \mathrm{~V}$ when Boltzmann electrons are assumed.

${ }^{\mathrm{i}}$ Temperatures reported for the stable double layer.

${ }^{\mathrm{j}}$ Bohm criterion for positive and negative ions.

mal drift velocity required between positive and negative ions in order to get two-stream instability [Eq. (15) in Ref. 37]. Interestingly, this criterion is verified at the position of each propagating double layer.

Note that it was shown by McKenzie ${ }^{50}$ that the Bohm criterion and Tuszewski's criterion are actually equivalent when the mass and the number of charges of the positive and negative ions are the same.

\section{PROPOSED MECHANISM FOR THE FORMATION OF PROPAGATING DOUBLE LAYERS}

The correlation of the data presented here allows to propose a mechanism for the formation of propagating double layers.

Propagating double layers are only observed (with the fully self-consistent simulation, Sec. IV) when a chamber of small diameter (no need of geometric transition) is combined with a localized heating mechanism (in the source region for example, from 0 to $15 \mathrm{~cm}$ ). Electron radial loss due to the small diameter has presumably three main effects. (i) The first and most obvious, observed under the present conditions of pressure (low pressure) is a depletion of the electron energy distribution function at energies higher than the plasma potential (Ref. 21, and references therein). As the depleted part of the distribution also corresponds to the ionizing electrons, this effect tends to decrease ionization. In response, the electron temperature (of the Maxwellian portion of the electron distribution) tends to increase to "boost" the ionization, to counterbalance the radial loss, and to maintain the plasma (fixed power).

(ii) The second effect of the electron radial loss, somewhat related to the first one, appears in particular when radial loss is combined with the localized heating mechanism. In such a case the electron temperature increases more in the heating region than in the rest of the discharge, hence leading to a nonuniformity of the electron temperature, with a higher temperature in the source than in the diffusion chamber. As a result of the electron temperature decrease, the source is ionization dominated (high temperature favoring ionization to attachment), while the diffusion chamber is attachmentdominated (lower temperature favoring attachment to ionization).

(iii) The third consequence of the electron radial loss becomes clear when combined with the ionizationdominated source. A significant fraction of the electrons created in the source are radially lost before being able to "fill up" the diffusion chamber, hence leading to a electron density profile and the associated plasma potential profile both decreasing from the source to the diffusion chamber.

To summarize, positive ions are essentially created in the source while negative ions are essentially created in the diffusion chamber. Due to the decrease of the plasma potential from the source to the diffusion chamber, positive and negative ions fall towards the diffusion chamber and the source, respectively. In other words, there exists two charged species drifting in opposite direction. For sufficiently 
long reactors, each species eventually reaches the Bohm velocity, breaking down the quasineutrality of the plasma and leading to the formation of an internal sheath (i.e., a double layer).

With such a mechanism, there is no reason that the formed double layers should remain in place as the system is highly nonsymmetrical; however, the reason the double layers propagate in one direction or the other remains unclear. We can postulate that because of a small charge unbalance within the double layers, there exists an internal force pushing them. Note that another mechanism should be sought for the stable double layer also observed by Plihon et al. ${ }^{22}$

\section{CONCLUSION}

The propagating double layers were experimentally characterized and the window of parameters, such as the neutral gas pressure, the relative concentration of $\mathrm{SF}_{6}$, the input power, etc., for which they form was fully determined in Ref. 23 . The propagating double layers spontaneously forming in the self-consistent simulation were shown to be a very similar phenomenon to that observed experimentally. The main features of the propagating double layers observed experimentally and in the simulation are summarized in Table II. Despite the fact that these propagating double layers do not propagate in the same direction, they share many properties, such as their velocity of propagation, frequency, electronegativity, temperature drop, etc. In addition, they appear under very similar conditions, such as neutral gas pressure, relative $\mathrm{SF}_{6}$ concentration, etc.

The simulation gave evidence supporting the observation that double layers form spontaneously in electronegative plasmas and allowed us to propose a formation mechanism by correlating the following observations. (i) To observe propagating double layers, both the experiment and the simulation have shown that a lower limit to the electronegativity is required, which is achieved thanks to a sufficient relative concentration of $\mathrm{SF}_{6}$ and sufficient attachment. (ii) The losses in volume of both positive and negative ions via recombination also appear to be crucial. The simulation showed that below a critical recombination rate, no double layer could be formed. (iii) The simulation also confirmed that the geometry of the chamber, i.e., a change in diameter, does not seem to be a critical parameter. (iv) However, the diameter itself is crucial, since it can dramatically change the electron energy distribution function that controls ionization and attachment and therefore the electronegativity. (v) The simulation showed that for chambers below a certain length, no double layer could be sustained, which is in good agreement with the experimental observations. This may well be because positive and negative charges need to develop a sufficient relative drift velocity, which can be achieved only in sufficiently long chambers. (vi) Finally, the neutral gas pressure is also a fundamental parameter, as no double layer was observed for pressure above $\sim 5 \mathrm{mTorr}$.

As a concluding remark, it may be noted that the propagating double layers of both the experiment and the simulation share many features with the downstream instability reported by Tuszewski et al. ${ }^{36}$ and Tuszewski and Gary. ${ }^{37}$
${ }^{1}$ M. A. Raadu, Phys. Rep. 178, 25 (1989).

${ }^{2}$ I. Langmuir, Phys. Rev. 33, 954 (1929).

${ }^{3}$ P. C. Stangeby and J. E. Allen, J. Phys. D 6, 224 (1973).

${ }^{4}$ N. Sato, G. Popa, E. Märk, E. Mravlag, and R. Schrittwieser, Phys. Fluids 19, 70 (1976).

${ }^{5}$ P. Coakley, N. Hershkowitz, R. Hubbard, and G. Joyce, Phys. Rev. Lett. 40, 230 (1978).

${ }^{6}$ C. Charles and R. W. Boswell, Appl. Phys. Lett. 82, 1356 (2003).

${ }^{7}$ H. Alfvén, Tellus 10, 104 (1958).

${ }^{8}$ R. D. Albert and P. J. Lindstrom, Science 170, 1398 (1970).

${ }^{9}$ M. Temerin, K. Cerny, W. Lotko, and F. S. Mozer, Phys. Rev. Lett. 48, 1175 (1982).

${ }^{10}$ H. Alfvén and P. Carlqvist, Sol. Phys. 1, 220 (1967).

${ }^{11}$ R. W. Boswell, E. Marsch, and C. Charles, Astrophys. J. 640, L199 (2006).

${ }^{12}$ S. A. Cohen, N. S. Siefert, S. Stange, E. E. Scime, and F. M. Levinton, Phys. Plasmas 10, 2593 (2003).

${ }^{13}$ O. Sutherland, C. Charles, N. Plihon, and R. W. Boswell, Phys. Rev. Lett. 95, 205002 (2005).

${ }^{14}$ A. M. Keesee, E. E. Scime, C. Charles, A. Meige, and R. W. Boswell, Phys. Plasmas 12, 093502 (2005).

${ }^{15}$ A. Aanesland, C. Charles, M. A. Lieberman, and R. W. Boswell, Phys. Rev. Lett. 97, 075003 (2006).

${ }^{16}$ N. Plihon, P. Chabert, and C. Corr, Phys. Plasmas 14, 013506 (2007).

${ }^{17}$ M. A. Lieberman and C. Charles, Phys. Rev. Lett. 97, 045003 (2006).

${ }^{18}$ A. Meige, R. W. Boswell, C. Charles, J.-P. Boeuf, G. J. M. Hagelaar, and M. M. Turner, IEEE Trans. Plasma Sci. 33, 334 (2005).

${ }^{19}$ A. Meige, R. W. Boswell, C. Charles, and M. M. Turner, Phys. Plasmas 12, 052317 (2005).

${ }^{20}$ X. Sun, A. M. Keesee, C. Biloiu, E. E. Scime, A. Meige, C. Charles, and R. W. Boswell, Phys. Rev. Lett. 95, 025004 (2005).

${ }^{21}$ A. Meige and R. W. Boswell, Phys. Plasmas 13, 092104 (2006).

${ }^{22}$ N. Plihon, C. S. Corr, and P. Chabert, Appl. Phys. Lett. 86, 091501 (2005).

${ }^{23}$ N. Plihon, C. S. Corr, P. Chabert, and J.-L. Raimbault, J. Appl. Phys. 98, 023306 (2005).

${ }^{24}$ I. G. Kouznetsov, A. J. Lichtenberg, and M. A. Lieberman, J. Appl. Phys. 86, 4142 (1999).

${ }^{25}$ T. E. Sheridan, J. Phys. D 32, 1761 (1999).

${ }^{26}$ T. E. Sheridan, P. Chabert, and R. W. Boswell, Plasma Sources Sci. Technol. 8, 457 (1999).

${ }^{27}$ T. E. Sheridan, N. S. J. Braithwaite, and R. W. Boswell, Phys. Plasmas 6, 4375 (1999).

${ }^{28}$ P. Chabert and T. E. Sheridan, J. Phys. D 33, 1854 (2000).

${ }^{29}$ J.-P. Boeuf, Phys. Rev. A 36, 2782 (1987).

${ }^{30}$ R. W. Boswell, Phys. Lett. 33A, 457 (1970).

${ }^{31}$ A. Cantin and R. R. J. Gagne, Appl. Phys. Lett. 30, 316 (1977).

${ }^{32}$ M. B. Hopkins and W. G. Graham, Rev. Sci. Instrum. 57, 2210 (1986).

${ }^{33}$ F. M. J. Gudmundsson, Tech. Rep., Science Institute University of Iceland, 2002.

${ }^{34}$ M. Bacal, Rev. Sci. Instrum. 71, 3981 (2000).

${ }^{35}$ M. Tuszewski and R. R. White, J. Appl. Phys. 94, 2858 (2003).

${ }^{36}$ M. Tuszewski, R. R. White, and G. A. Wurden, Plasma Sources Sci. Technol. 12, 396 (2003).

${ }^{37}$ M. Tuszewski and S. Gary, Phys. Plasmas 10, 539 (2003).

${ }^{38}$ R. W. Hockney and J. W. Eastwood, Computer Simulation Using Particles (IOP, Bristol, 1988).

${ }^{39}$ C. K. Birdsall and A. B. Langdon, Plasma Physics via Computer (McGraw-Hill, New York, 1985).

${ }^{40}$ A. Meige, Ph.D. thesis, The Australian National University (Canberra) and Université Paul Sabatier (Toulouse), 2006.

${ }^{41}$ W. H. Press, S. A. Teukolsky, W. T. Vetterling, and B. P. Flannery, Numerical Recipes in C: The Art of Scientific Computing (Cambridge University Press, Cambridge, 1992).

${ }^{42}$ G. J. M. Hagelaar, "How to normalize the density of Boltzmann electrons in a transient self-consistent plasma model" (unpublished).

${ }^{43}$ L. Garrigues, A. Heron, J. C. Adam, and J. P. Boeuf, Plasma Sources Sci. Technol. 9, 219 (2000).

${ }^{44}$ P. L. G. Ventzek, T. J. Sommerer, R. J. Hoekstra, and M. J. Kushner, Appl. Phys. Lett. 63, 605 (1993).

${ }^{45}$ D. T. K. Kwok, Z. M. Zeng, P. K. Chu, and T. E. Sheridan, J. Phys. D 34, 1091 (2001).

${ }^{46}$ A. V. Phelps and R. J. van Brunt, J. Appl. Phys. 64, 4269 (1988). 
${ }^{47}$ C. M. Ferreira, G. Gousset, and M. Touzeau, J. Phys. D 21, 1403 (1988).

${ }^{48}$ N. S. J. Braithwaite and J. E. Allen, J. Phys. D 21, 1733 (1988).

${ }^{49}$ R. N. Franklin and J. Snell, J. Phys. D 25, 453 (1992).

${ }^{50}$ J. F. McKenzie, J. Plasma Phys. 65, 181 (2001).

${ }^{51}$ Here, the term "temperature" is used loosely, as the EEDF may not be
Maxwellian. What is meant by "temperature" is "the temperature that would have a Maxwellian distribution with the same mean energy."

${ }^{52}$ Reaction rate coefficients $k$ can generally be well fitted by a generalized Arrhenius function of the temperature $T_{e}$, of the form $k\left(T_{e}\right)=\exp (a$ $\left.+b \ln T_{e}+c / T_{e}+d / T_{e}^{2}+e / T_{e}^{3}\right)$. 